\title{
EDITORIAL
}

\section{MAMOGRAFÍA Y DETECCIÓN PRECOZ DEL CÁNCER DE MAMA}

\author{
José Asua Batarrita \\ Jefe del Plan de Salud de Euskadi. Dirección Territorial de Sanidad de Bizkaia. Departamento de Sanidad, Gobierno \\ Vasco.
}

El cáncer de mama supone la causa de muerte más frecuente entre las mujeres de los países desarrollados. Su prevención primaria es por ahora un interrogante, aunque sí se pueden hacer esfuerzos para realizar, al menos, una prevención secundaria o diagnóstico precoz.

La mamografía se plantea como la técnica de diagnóstico de cáncer de mama más utilizada universalmente. Durante las últimas dos décadas se han venido implantando programas de base poblacional en distintos países, en ocasiones a nivel municipal o de regiones, como fue el caso de Suecia o de algunas zonas de EEUU. En nuestro contexto más cercano fue Navarra la primera Comunidad Autónoma en poner en marcha un programa de cobertura poblacional en 1990. A partir de entonces durante esa década se desarrollaron estudios específicos que supusieron que prácticamente todas las Comunidades Autónomas (CCAA) ${ }^{1}$ pusieran en marcha programas de detección, aunque no todos alcanzan el $100 \%$ de cobertura o una participación elevada.

Correspondencia:

José Asua Batarrita

Plan de Salud de Euskadi

Dirección Territorial de Sanidad de Bizkaia

Departamento de Sanidad, Gobierno Vasco

Gran Vía $81-1^{\circ}$

48011 Bilbao

Correo electrónico: plsabi-san@ej-gv.es
El efecto de estos programas puede observarse desde diferentes perspectivas. En primer lugar, gracias a la dinámica de captación que conllevan han conseguido que desaparezcan las diferencias anteriormente existentes en la utilización de la mamografía entre las mujeres de los distintos niveles socioeconómicos, como se ha podido ver al analizar las sucesivas Encuestas de Salud realizadas en el País Vasco².

En cuanto a la carga de la enfermedad que supone el cáncer de mama, mientras su incidencia aumentó un 0,3 por año entre 1987 y 2002 , las tasas de mortalidad han disminuido un $2,3 \%$ por año desde $1990,3,3 \%$ entre las mujeres menores de 50 y $2,0 \%$ por año en las mujeres de 50 años o más.

Parece que con la implantación de los programas de detección precoz se cumplen parte de los objetivos planteados desde el inicio: aumentar la calidad y la esperanza de vida de las mujeres con cáncer de mama.

Durante los primeros años de funcionamiento de los programas, como era lógico y esperable, la mayor actividad de búsqueda de tumores entrañaba que se diagnostiquen en mayor número y en estadios más tempranos, lo que se ha reflejado en un incremento de la incidencia. Pero, paralelamente, gracias a las nuevas técnicas curativas se ha conseguido disminuir la mortalidad por esta 
causa y alcanzar un mejor pronóstico e incrementar de esta manera la calidad de vida de las mujeres afectadas.

Aún así, quedan algunas cuestiones en torno a los aspectos organizativos, estrategias y poblaciones a las que dirigirse o periodicidad de las pruebas, que de forma repetida generan temas de discusión y son fruto de diferentes investigaciones.

Actualmente, nadie parece dudar que la periodicidad más conveniente es realizar mamografías de forma bienal (cada dos años). Asimismo, existe consenso sobre la realización de doble proyección por cada mama. Sin embargo, una de las cuestiones más frecuentemente debatida se refiere a los grupos de edad a los que debe dirigirse esta actividad preventiva. En España ${ }^{3}$ existen programas que comienzan a partir de los 45 y otros a partir de los 50 años, hasta los que alcanzan a mujeres hasta los 64, 65, 69 y alguna CCAA incluso hasta los 70 años.

Se observa que un buen número de CCAA ha decidido elevar el tramo superior de las edades de las mujeres citadas a los programas. Así durante los últimos años se va incrementando de forma paulatina esta franja de edad.

Como puede verse en la tabla 1, en los diferentes programas autonómicos ${ }^{4}$ se ha

Tabla 1

\begin{abstract}
Descripción de las principales características de los programas de detección precoz del cáncer de mama por Comunidad Autónoma
\end{abstract}

\begin{tabular}{|c|c|c|c|c|c|}
\hline Comunidad Autónoma & Año Inicio & $\begin{array}{c}\text { Población } \\
\text { Diana }\end{array}$ & $\begin{array}{c}\text { \% Población } \\
\text { Cubierta }\end{array}$ & \% Participación & $\begin{array}{l}\text { Unidades de } \\
\text { Exploración }\end{array}$ \\
\hline Andalucía & 1995 & $50-65$ & 100 & 64,14 & 44 \\
\hline Aragón & 1997 & $50-64$ & 86 & -- & 6 \\
\hline Asturias & 1998 & $50-65$ & 100 & -- & 8 \\
\hline Baleares & 1997 & $50-64$ & 74 & -- & 6 \\
\hline Canarias & 1999 & $50-67$ & 92,54 & 62,28 & 12 \\
\hline Cantabria & 1997 & $50-64$ & 100 & 57,26 & 2 \\
\hline Castilla y León & 1992 & $45-70$ & 100 & 67,30 & 12 \\
\hline Castilla-La Mancha & 1992 & $45-69$ & 100 & 61,06 & 8 \\
\hline Cataluña & 1992 & $50-69$ & 100 & 57,55 & 57 \\
\hline Valencia & 1992 & $45-69$ & 100 & 73,32 & 24 \\
\hline Extremadura & 1998 & $50-64$ & 93,47 & 56,99 & 9 \\
\hline Galicia & 1992 & $50-64$ & 100 & 72,86 & 10 \\
\hline Madrid & 1998 & $50-64$ & 100 & 29,47 & 6 \\
\hline Murcia & 1994 & $50-69$ & 100 & 71,19 & 5 \\
\hline Navarra & 1990 & $45-69$ & 100 & 87,88 & 2 \\
\hline País Vasco & 1995 & $50-64$ & 100 & 79,59 & 9 \\
\hline La Rioja & 1993 & $45-65$ & 100 & -- & 2 \\
\hline
\end{tabular}

Elaboración propia a partir de: http://www.programascancerdemama.org 
alcanzado una participación que va desde el $87,88 \%$ en Navarra y el 79,59\% en País Vasco al 29,47\% de Madrid, si bien el resto de CCAA alcanzan una cifra en torno al $60 \%$. En cuanto a la adherencia, el promedio en general se sitúa en torno al $85 \%$ y la detección alrededor del $10-15 \%$ de tumores in situ.

Mucho se ha escrito sobre esta cuestión, aunque una de las últimas revisiones sistemáticas de la bibliografía realizadas sobre este tema ${ }^{5}$ zanja definitivamente el debate asegurando que, según la información científica disponible, la mayor reducción de la mortalidad gracias a un programa de detección precoz se da en las mujeres a partir de los 50 años. Asimismo, recomienda que los programas de detección alcancen a las mujeres de hasta 69 años.

El artículo presentado en este número de la revista ${ }^{6}$ aborda un importante aspecto del éxito de un programa de detección precoz, como son los factores que condicionan la incorporación y adherencia de las mujeres a las pruebas ofrecidas. Se observa que las mujeres jóvenes y las de menor edad incluidas en los programas de detección son las que muestran en mayor medida actitudes positivas y un mayor nivel de información con respecto a la mamografía en la encuesta de 2000 frente a la realizada en 1994.

Si bien las coberturas de los programas autonómicos han ido elevándose de manera que 13 CCAA llegan ya al $100 \%$, quedan aún esfuerzos por hacer para alcanzar participaciones deseables en torno al 75-80\%. La aportación de este estudio supone una contribución para ahondar en el conocimiento de los factores que influyen en la participación en los programas y, de esta forma, ayudará a diseñar estrategias para su mejora.

De todas formas, otras intervenciones están influyendo de manera positiva en el diagnóstico y pronóstico del cáncer de mama ${ }^{7}$. Así, desde hace unos años la ayuda que representa disponer de mamotomos con biopsia estereotáxica puede suponer un ahorro de hasta el $58 \%$ con respecto a la biopsia quirúrgica y la realizada mediante biopsia percutánea escisional $(\mathrm{ABBI})^{8}$ presenta un coste casi $48 \%$ menor, aunque ello suponga una importante inversión inicial en la adquisición de estas nuevas técnicas. Asimismo, se ha podido comprobar una importante disminución de biopsias quirúrgicas con el consiguiente beneficio para las mujeres.

Por otra parte, sin duda a medio plazo, estarán accesibles (y esperemos a un coste razonable) nuevas técnicas de imagen que pueden superar la calidad del mamógrafo ${ }^{9}$. Así, hace tiempo que está comercializada la prometedora mamografía digital, aunque todavía no está suficientemente difundida. Esta técnica implica evitar placas de celuloide, reveladoras y voluminosos (y engorrosos) sistemas de archivo, aunque exigirá mayor y mejor dotación informática y un aprendizaje o adaptación por parte del personal clínico de las unidades detección.

En una perspectiva más de futuro (aunque quizás no muy lejano) artículos recientes afirman que los biochips de ADN ayudarán a identificar distintos tipos de cáncer de mama, aunque en este caso se refieren sobre todo a tumores de mama invasivos.

\section{BIBLIOGRAFÍA}

1. Asua J, Rico R, Gutiérrez MA, Aranáez R. Detección precoz del cáncer de mama en la CAPV. Propuesta de actuación. Vitoria-Gasteiz: Departamento de Sanidad, Gobierno Vasco, Osteba; 1994.

2. Gobierno Vasco. Encuesta de Salud de la CA del País Vasco 2002. Vitoria-Gasteiz: Servicio de publicaciones del Gobierno Vasco; 2004.

3. Luengo S, Muñoz A. Uso de la mamografía y de la citología de Papanicolaou para la detección precoz del cáncer de mama y de cérvix uterino en España. Madrid: Agencia de Evaluación de Tecnologías Sanitarias, Instituto de Salud Carlos III; 2002. 
4. Programas de cáncer de mama. Disponible en: http://www.programascancerdemama.org.

5. Deck W, Kakuma R. Mammographie de dépistage: une réévaluation. Québec: AETMIS;2005.

6. Luengo Matos S, Polo de Santos MM. Diferencias en los factores relacionados con el uso de la mamografía en las mujeres españolas en dos encuestas realizadas en 1994 y 2000 . Rev Esp Salud Pública 2005.

7. Jausoro A. Nuevas técnicas de diagnóstico del cáncer de mama. Vitoria-Gasteiz: Departamento de Sanidad, Gobierno Vasco, Osteba; 2000.
8. Gutiérrez A, Taboada J, Apesteguia L, Arrizabalaga R, Asua J, Andreu FJ. Nuevas técnicas percutáneas de diagnóstico histológico de lesiones no palpables por sospecha de cáncer de mama. Proyecto FIS, Vitoria-Gasteiz, Departamento de Sanidad, Gobierno Vasco, 2005 Informe número Osteba D05-02.

9. Pisano ED, Gatsonis C, Hendrick E, Yaffe M, Baum JK, Acharyya S, Conant EF, Fajardo LL, Bassett L, D'Orsi C, Jong R, Rebner M. Diagnostic Performance of Digital versus Film Mammography for Breast-Cancer Screening. N Engl J Med 2005. 